\title{
RECOGNIZING RIGHT-LEFT EQUIVALENCE LOCALLY
}

\author{
TAKASHI NISHIMURA \\ Department of Mathematics, Faculty of Education and Human Sciences \\ Yokohama National University \\ Yokohama 240-8501, Japan \\ E-mail: takashi@edhs.ynu.ac.jp
}

The present paper is a survey of the author's recent results on recognizing $C^{r}$ right-left equivalence of $C^{\infty}$ map-germs $(0 \leq r \leq \infty)$.

We say two $C^{\infty}$ map-germs are $C^{r}$ right-left equivalent if they coincide under germs of appropriate $C^{r}$ co-ordinate systems of the source space and the target space, where a $C^{0}$ co-ordinate system means a co-ordinate system given by a homeomorphism. We often encounter the situations where we would like to decide whether or not given two map-germs are $C^{r}$ right-left equivalent. In the case that one of them is of full rank (resp. linear), the implicit function theorem (resp. the rank theorem) answers our purpose (possibly except for $r=0$ ). However, how can we decide in general case? By using a simple systematic method explained in Section 4, we can obtain many results to the problem. In Section 1, we give a series of criteria for $C^{r}$ right-left equivalence of $C^{\infty}$ map-germs $(1 \leq r \leq \infty)$. In Section 2, infinitesimal refinements of criteria for $C^{\infty}$ rightleft equivalence of $C^{\infty}$ map-germs are given. Next, we consider $C^{0}$ right-left equivalence. In Section 3, we give a series of criteria for $C^{0}$ right-left equivalence of $\mathcal{K}$-equivalent map-germs. All of the results are derived from one simple idea, which is the key of our systematic method and explained exhaustively in Section 4. In Section 5 we give several applications of our results, which show how useful our method is.

The results for $r=\infty$ are all valid both in the real analytic category and in the complex analytic category as well.

1. Criteria for $C^{r}$ right-left equivalence $(1 \leq r \leq \infty)$. For a given $C^{\infty}$ mapgerm $f:\left(\mathbf{R}^{n}, 0\right) \rightarrow\left(\mathbf{R}^{p}, 0\right)$, any $C^{r}$ map-germ $\Phi:\left(\mathbf{R}^{n} \times \mathbf{R}^{k},(0,0)\right) \rightarrow\left(\mathbf{R}^{p}, 0\right)$ such that $\Phi(x, 0)=f(x)$ is called a $C^{r}$ deformation-germ of $f$. A $C^{r}$ deformation-germ $\Phi:\left(\mathbf{R}^{n} \times \mathbf{R}^{k},(0,0)\right) \rightarrow\left(\mathbf{R}^{p}, 0\right)$ of $f:\left(\mathbf{R}^{n}, 0\right) \rightarrow\left(\mathbf{R}^{p}, 0\right)$ is said to be $C^{r}$-trivial if

1991 Mathematics Subject Classification: Primary 58C27; Secondary 14B05, 32S30.

The paper is in final form and no version of it will be published elsewhere. 
there exist germs of $C^{r}$ diffeomorphisms $h:\left(\mathbf{R}^{n} \times \mathbf{R}^{k},(0,0)\right) \rightarrow\left(\mathbf{R}^{n} \times \mathbf{R}^{k},(0,0)\right)$ and $H:\left(\mathbf{R}^{p} \times \mathbf{R}^{k},(0,0)\right) \rightarrow\left(\mathbf{R}^{p} \times \mathbf{R}^{k},(0,0)\right)$ such that the following diagram (*) commutes, where $\pi:\left(\mathbf{R}^{n} \times \mathbf{R}^{k},(0,0)\right) \rightarrow\left(\mathbf{R}^{k}, 0\right), \quad \pi^{\prime}:\left(\mathbf{R}^{p} \times \mathbf{R}^{k},(0,0)\right) \rightarrow\left(\mathbf{R}^{k}, 0\right)$, are canonical projections:

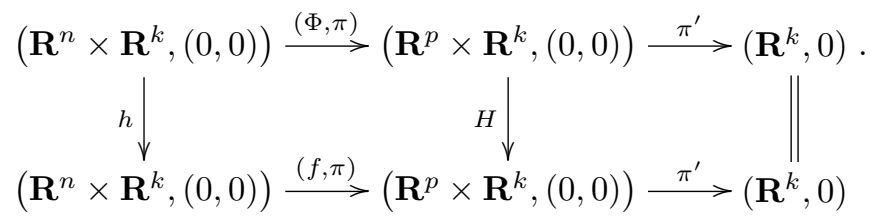

For given two $C^{\infty}$ map-germs $f, g:\left(\mathbf{R}^{n}, 0\right) \rightarrow\left(\mathbf{R}^{p}, 0\right)$, we consider the following conditions $\left(\mathrm{i}_{\mathrm{r}}\right),\left(\mathrm{ii}_{\mathrm{r}}\right),\left(\mathrm{iii} \mathrm{r}_{\mathrm{r}}\right)$ and $\left(\mathrm{iv}_{\mathrm{r}}\right)$.

$\left(\mathrm{i}_{\mathrm{r}}\right)$ The map-germ $f$ is $C^{r}$ right-left equivalent to $g$.

$\left(\mathrm{ii}_{\mathrm{r}}\right)$ There exist a germ of $C^{r}$ diffeomorphism $s:\left(\mathbf{R}^{n}, 0\right) \rightarrow\left(\mathbf{R}^{n}, 0\right)$ and a $C^{r}$ map-germ $M:\left(\mathbf{R}^{n}, 0\right) \rightarrow(G L(p, \mathbf{R}), M(0))$ such that the following (a) and (b) are satisfied:

(a) $f(x)=M(x) g(s(x))$,

(b) the $C^{r}$ map-germ $F:\left(\mathbf{R}^{n} \times \mathbf{R}^{p},(0,0)\right) \rightarrow\left(\mathbf{R}^{p}, 0\right)$ given by

$$
F(x, \lambda)=f(x)-M(x) \lambda
$$

is a $C^{r}$-trivial deformation-germ of $f$.

(iiii $\left.{ }_{r}\right)$ There exist a germ of $C^{r}$ diffeomorphism $s:\left(\mathbf{R}^{n}, 0\right) \rightarrow\left(\mathbf{R}^{n}, 0\right)$ and a $C^{r}$ map-germ $M:\left(\mathbf{R}^{n}, 0\right) \rightarrow(G L(p, \mathbf{R}), M(0))$ such that (a), (b) of condition (ii $\left.{ }_{\mathrm{r}}\right)$ and the following (c) are satisfied:

(c) The $C^{r}$ map-germ $G:\left(\mathbf{R}^{n} \times \mathbf{R}^{p},(0,0)\right) \rightarrow\left(\mathbf{R}^{p}, 0\right)$ given by

$$
G(x, \lambda)=g(x)-M\left(s^{-1}(x)\right)^{-1} \lambda
$$

is a $C^{r}$-trivial deformation-germ of $g$.

$\left(\mathrm{iv}_{\mathrm{r}}\right)$ There exist a germ of $C^{r}$ diffeomorphism $s:\left(\mathbf{R}^{n}, 0\right) \rightarrow\left(\mathbf{R}^{n}, 0\right)$ and a $C^{r}$ map-germ $M:\left(\mathbf{R}^{n}, 0\right) \rightarrow(G L(p, \mathbf{R}), M(0))$ such that (a), (b) of condition (ii $\left.{ }_{r}\right)$ and the following (d) are satisfied:

(d) The germ $\left(H\left(\{0\} \times \mathbf{R}^{p}\right), 0\right)$ is transverse to the germ $\left(\{0\} \times \mathbf{R}^{p}, 0\right)$, where $H$ is the germ of $C^{r}$ diffeomorphism of $\left(\mathbf{R}^{p} \times \mathbf{R}^{p}, 0\right)$ given in the above commutative diagram $(*)$ with $k, \Phi$ replaced by $p, F$.

First, we consider rank zero cases.

Theorem 1.1 ([15]). Let $f, g:\left(\mathbf{R}^{n}, 0\right) \rightarrow\left(\mathbf{R}^{p}, 0\right)$ be $C^{\infty}$ map-germs with rank zero. Then condition $\left(\mathrm{ii}_{\mathrm{r}}\right.$ ) implies condition $\left(\mathrm{i}_{\mathrm{r}}\right)$ for $1 \leq r \leq \infty$.

Next, we consider positive rank cases.

Example 1.1. Let $f, g:\left(\mathbf{R}^{2}, 0\right) \rightarrow\left(\mathbf{R}^{2}, 0\right)$ be given by

$$
\begin{aligned}
& f(x, y)=\left(x, y^{3}+x y\right), \\
& g(x, y)=\left(x, y^{3}\right)
\end{aligned}
$$


and $M:\left(\mathbf{R}^{2}, 0\right) \rightarrow\left(G L(2, \mathbf{R}), E_{2}\right)$ be given by

$$
M(x, y)=\left[\begin{array}{ll}
1 & 0 \\
y & 1
\end{array}\right]
$$

where $E_{2}$ is the unit 2 by 2 matrix. Then $f(x, y)=M(x, y) g(x, y)$.

It is well known that any $C^{\infty}$ deformation-germ of the map-germ $f$ is $C^{\infty}$-trivial. Thus, $\left(\mathrm{ii}_{\infty}\right)$ is satisfied. However, for any $1 \leq r \leq \infty$ condition $\left(\mathrm{i}_{\mathrm{r}}\right)$ does not hold (in fact, $f$ and $g$ are even not topologically right-left equivalent).

This example shows that condition ( $\left.\mathrm{ii}_{\mathrm{r}}\right)$ does not necessarily imply condition $\left(\mathrm{i}_{\mathrm{r}}\right)$ in positive rank cases. Nevertheless, the following holds under no assumptions.

Theorem $1.2([15])$. Condition (iii $\mathrm{r}_{\mathrm{r}}$ ) implies condition $\left(\mathrm{i}_{\mathrm{r}}\right)$ for $1 \leq r \leq \infty$.

Although Theorem 1.2 is interesting in itself, we prefer the $C^{r}$ triviality of the linearly parametrized deformation-germ of only one of $f$ or $g$ to those of both of $f$ and $g$. Thus, we are led to condition $\left(\mathrm{iv}_{\mathrm{r}}\right)$.

TheOrem 1.3 ([15]). Condition (iv $\mathrm{v}_{\mathrm{r}}$ ) implies condition $\left(\mathrm{i}_{\mathrm{r}}\right)$ for $1 \leq r \leq \infty$.

In the case $r=\infty$, we have

Theorem 1.4 ([15]). For any $C^{\infty}$ map germs $f, g:\left(\mathbf{R}^{n}, 0\right) \rightarrow\left(\mathbf{R}^{p}, 0\right)$, the following hold:

(1) $\quad\left(\mathrm{i}_{\infty}\right) \Leftrightarrow\left(\mathrm{iii}_{\infty}\right) \Leftrightarrow\left(\mathrm{iv}_{\infty}\right)$.

(2) $\quad\left(\mathrm{i}_{\infty}\right) \Leftrightarrow\left(\mathrm{ii}_{\infty}\right) \Leftrightarrow\left(\mathrm{iii}_{\infty}\right) \Leftrightarrow\left(\mathrm{iv}_{\infty}\right)$ if the rank of $f$ is zero.

Therefore, we may answer the $C^{\infty}$ recognition problem completely by using our conditions in principle.

2. Infinitesimal refinements of criteria for $C^{\infty}$ right-left equivalence. First, we review infinitesimal notations briefly. For details on them, see [9], [14], [15], [21].

Let $\mathcal{E}_{n}$ (resp. $\left.m_{n}\right)$ denote the set of $C^{\infty}$ function-germs $\left(\mathbf{R}^{n}, 0\right) \rightarrow \mathbf{R}$ (resp. $\left(\mathbf{R}^{n}, 0\right) \rightarrow$ $(\mathbf{R}, 0))$. The set $\mathcal{E}_{n}$ has a natural $\mathbf{R}$-algebra structure and the set $m_{n}$ is the unique maximal ideal in $\mathcal{E}_{n}$. For any positive integer $\ell, m_{n}^{\ell}$ means the $\ell$-times product of $m_{n}$. For $\ell=0, m_{n}^{0}$ is $\mathcal{E}_{n}$.

For a $C^{\infty}$ map-germ $f:\left(\mathbf{R}^{n}, 0\right) \rightarrow\left(\mathbf{R}^{p}, 0\right)$, let $\theta(f)$ denote the $\mathcal{E}_{n}$-module consisting of germs of $C^{\infty}$ vector fields $\zeta:\left(\mathbf{R}^{n}, 0\right) \rightarrow T\left(\mathbf{R}^{p}\right)$ such that $\pi_{p} \circ \zeta=f$, where $\pi_{p}: T\left(\mathbf{R}^{p}\right) \rightarrow \mathbf{R}^{p}$ denotes the canonical projection. By using the standard identification of $T\left(\mathbf{R}^{p}\right)$ with $\mathbf{R}^{p} \times \mathbf{R}^{p}, \theta(f)$ may be identified with the free $\mathcal{E}_{n}$-module with $p$-generators. When the given $f$ is the identity map-germ $\left(\mathbf{R}^{n}, 0\right) \rightarrow\left(\mathbf{R}^{n}, 0\right), \theta(f)$ may be denoted by $\theta(n)$.

For a $C^{\infty}$ map-germ $f:\left(\mathbf{R}^{n}, 0\right) \rightarrow\left(\mathbf{R}^{p}, 0\right)$, let $T f:\left(T \mathbf{R}^{n}, \pi_{n}^{-1}(0)\right) \rightarrow\left(T \mathbf{R}^{p}, \pi_{p}^{-1}(0)\right)$ denote the tangent map-germ of $f$, where $\pi_{n}: T \mathbf{R}^{n} \rightarrow \mathbf{R}^{n}, \pi_{p}: T \mathbf{R}^{p} \rightarrow \mathbf{R}^{p}$ are canonical projections. We define

$$
t f: \theta(n) \rightarrow \theta(f), \quad w f: \theta(p) \rightarrow \theta(f)
$$


by $t f(\xi)=T f \circ \xi, w f(\eta)=\eta \circ f$. By using $t f$ and $w f$, we define

$$
\begin{aligned}
T \mathcal{A}(f) & =t f\left(m_{n} \theta(n)\right)+w f\left(m_{p} \theta(p)\right) \quad \text { and } \\
T_{e} \mathcal{A}(f) & =t f(\theta(n))+w f(\theta(p)) .
\end{aligned}
$$

Theorem 2.1. Let $f, g:\left(\mathbf{R}^{n}, 0\right) \rightarrow\left(\mathbf{R}^{p}, 0\right)$ be $C^{\infty}$ map-germs with rank zero. Suppose that there exist a germ of $C^{\infty}$ diffeomorphism $s:\left(\mathbf{R}^{n}, 0\right) \rightarrow\left(\mathbf{R}^{n}, 0\right)$ and a $C^{\infty}$ map-germ $M:\left(\mathbf{R}^{n}, 0\right) \rightarrow(G L(p, \mathbf{R}), M(0))$ such that $f(x)=M(x) g(s(x))$. Suppose furthermore that there exists an integer $k \quad(k \geq 0)$ such that

(a) each entry of $M-M(0)$ belongs to $m_{n}^{k+1}$ and

(b) $m_{n}^{k} \theta(f) \subset T_{e} \mathcal{A}(f)$.

Then $f$ and $g$ are $C^{\infty}$ right-left equivalent.

Although there are no proofs of Theorem 2.1 in a series of author's papers [13]-[18], by the proof of Theorem 2.2 below it is clear that conditions (a) and (b) of Theorem 2.1 imply the $C^{\infty}$-triviality of $f(x)-M(x) \lambda$. Thus, Theorem 2.1 follows from Theorem 1.1.

Theorem 2.2 ([14]). Let $f, g:\left(\mathbf{R}^{n}, 0\right) \rightarrow\left(\mathbf{R}^{p}, 0\right)$ be $C^{\infty}$ map-germs. Suppose that there exist a germ of $C^{\infty}$ diffeomorphism $s:\left(\mathbf{R}^{n}, 0\right) \rightarrow\left(\mathbf{R}^{n}, 0\right)$ and a $C^{\infty}$ map-germ $M:\left(\mathbf{R}^{n}, 0\right) \rightarrow(G L(p, \mathbf{R}), M(0))$ such that $f(x)=M(x) g(s(x))$. Suppose furthermore that there exists an integer $k \quad(k \geq 0)$ such that

(a) each entry of $M-M(0)$ belongs to $m_{n}^{k+1}$,

(b) $m_{n}^{k} \theta(f) \subset T_{e} \mathcal{A}(f)$ and

(c) $m_{n}^{k} \theta(g) \subset T_{e} \mathcal{A}(g)$.

Then $f$ and $g$ are $C^{\infty}$ right-left equivalent.

Theorem 2.2 was stated (but not proved) first by A. A. du Plessis ([19], page 128). Conditions (a) and (b) (resp. (a) and (c)) of Theorem 2.2 imply the $C^{\infty}$-triviality of $f(x)-M(x) \lambda$ (resp. $\left.g(x)-M\left(s^{-1}(x)\right)^{-1} \lambda\right)$. Thus, Theorem 2.2 follows from Theorem 1.2 (for details, see [14]).

Theorem $2.3([14])$. Let $f, g:\left(\mathbf{R}^{n}, 0\right) \rightarrow\left(\mathbf{R}^{p}, 0\right)$ be $C^{\infty}$ map-germs. Suppose that there exist a germ of $C^{\infty}$ diffeomorphism $s:\left(\mathbf{R}^{n}, 0\right) \rightarrow\left(\mathbf{R}^{n}, 0\right)$ and a $C^{\infty}$ map-germ $M:\left(\mathbf{R}^{n}, 0\right) \rightarrow(G L(p, \mathbf{R}), M(0))$ such that $f(x)=M(x) g(s(x))$. Suppose furthermore that there exists a positive integer $k$ such that

(a) each entry of $M-M(0)$ belongs to $m_{n}^{k}$ and

(b) $m_{n}^{k} \theta(f) \subset T \mathcal{A}(f)$.

Then $f$ and $g$ are $C^{\infty}$ right-left equivalent.

Conditions (a) and (b) imply condition $\left(\mathrm{iv}_{\infty}\right)$. Thus Theorem 2.3 follows from Theorem 1.3 (for details, see [14]). Although we can deduce infinitesimal results from Theorem 1.3 in a different way, Theorem 2.3 is the most standard infinitesimal refinement of Theorem 1.3 and quite useful as shown in the following examples and $§ 5.3$. 
EXAMPLE 2.1 (taken from [12]). This example is almost the same as Example 1.1 in [14]. Let $f:\left(\mathbf{R}^{2}, 0\right) \rightarrow\left(\mathbf{R}^{3}, 0\right)$ be given by

$$
f(x, y)=\left(x, x y+y^{3}, x y^{2}+y^{10}\right) .
$$

Thanks to D. Mond, the following have been known as information on $f([12]$, Theorem 4.2.2:7).

(2.1.1) The map-germ $f$ is 10 -determined with respect to $C^{\infty}$ right-left equivalence.

(2.1.2) The map-germ $f$ is not 9-determined with respect to $C^{\infty}$ right-left equivalence.

(2.1.3) $m_{2}^{8} \theta(f)$ is contained in $T \mathcal{A}(f)$.

Let $N(x, y)$ be a 3 by 3 matrix with entries belonging to $m_{2}^{8}$. Then, by Theorem 2.3, $g=f+N f$ is $C^{\infty}$ right-left equivalent to $f$. In fact, $f=\left(E_{3}+N\right)^{-1} g$ and $M=\left(E_{3}+N\right)^{-1}$ satisfies $M(0)=E_{3}$ and each entry of $M-M(0)$ belongs to $m_{2}^{8}$.

EXAMPLE 2.2 (taken from $[6])$. Let $f:\left(\mathbf{R}^{2}, 0\right) \rightarrow\left(\mathbf{R}^{3}, 0\right)$ be given by

$$
f(x, y)=\left(x, y^{3}+x^{2} y, y^{4}+x y^{2}\right) .
$$

Thanks to T. Gaffney and A. A. du Plessis, the following has been known as one of information on $f([6]$, Example (3.6)):

$$
m_{2}^{3} \theta(f) \subset T \mathcal{A}(f) .
$$

Let $N(x, y)$ be a 3 by 3 matrix with entries belonging to $m_{2}^{3}$. Then, by Theorem 2.3 , $g=f+N f$ is $C^{\infty}$ right-left equivalent to $f$. In fact, $f=\left(E_{3}+N\right)^{-1} g$ and $M=$ $\left(E_{3}+N\right)^{-1}$ satisfies $M(0)=E_{3}$ and each entry of $M-M(0)$ belongs to $m_{2}^{3}$. Combining this result with direct co-ordinate manipulations yields the same $M$-determinacy result as in Example (3.6) of [6]. Note that the only information which we require is (2.2.1).

3. Criteria for $C^{0}$ right-left equivalence. A $C^{\infty}$ deformation-germ $\Phi:\left(\mathbf{R}^{n} \times\right.$ $\left.\mathbf{R}^{k},(0,0)\right) \rightarrow\left(\mathbf{R}^{p}, 0\right)$ of $f$ is said to be Thom trivial (resp. transversely Thom trivial) if there exist C-regular stratifications in the sense of Bekka ([2]), $\mathcal{S}$ of $\mathbf{R}^{n} \times \mathbf{R}^{k}, \mathcal{T}$ of $\mathbf{R}^{p} \times \mathbf{R}^{k}$ and $\left\{\mathbf{R}^{k}\right\}$ of $\mathbf{R}^{k}$ such that the following (T1) and (T2) (resp. (T1), (T2) and (T3)) hold:

(T1) The map-germ

$$
(\Phi, \pi):\left(\mathbf{R}^{n} \times \mathbf{R}^{k},(0,0)\right) \rightarrow\left(\mathbf{R}^{p} \times \mathbf{R}^{k},(0,0)\right)
$$

is a Thom map-germ with respect to $\mathcal{S}$ and $\mathcal{T}$.

(T2) The map-germ

$$
\pi^{\prime}:\left(\mathbf{R}^{p} \times \mathbf{R}^{k},(0,0)\right) \rightarrow\left(\mathbf{R}^{k}, 0\right)
$$

is a stratified map-germ (or equivalently in this case, a Thom map-germ) with respect to $\mathcal{T}$ and $\left\{\mathbf{R}^{k}\right\}$.

(T3) The stratum $T_{0}$ of $\mathcal{T}$, which contains the origin $(0,0)$ of $\mathbf{R}^{p} \times \mathbf{R}^{k}$, is transverse to $\{0\} \times \mathbf{R}^{k}\left(\subset \mathbf{R}^{p} \times \mathbf{R}^{k}\right)$.

Here $\pi:\left(\mathbf{R}^{n} \times \mathbf{R}^{k},(0,0)\right) \rightarrow\left(\mathbf{R}^{k}, 0\right), \pi^{\prime}:\left(\mathbf{R}^{p} \times \mathbf{R}^{k},(0,0)\right) \rightarrow\left(\mathbf{R}^{k}, 0\right)$ are canonical projections. For the definition of a C-regular stratification, see [2]. We remark that the notion of a C-regular stratification is an extended one of a Whitney stratification and it 
is known that every C-regular stratification admits a controlled tube system ([2]). For the definitions of a stratified map-germ and a Thom map-germ, see [2], [7], [11].

By Thom's second isotopy lemma ([2], [7], [11]), we see that for any Thom trivial deformation-germ $\Phi:\left(\mathbf{R}^{n} \times \mathbf{R}^{k},(0,0)\right) \rightarrow\left(\mathbf{R}^{p}, 0\right)$ of $f$, there exist germs of homeomorphisms $h:\left(\mathbf{R}^{n} \times \mathbf{R}^{k},(0,0)\right) \rightarrow\left(\mathbf{R}^{n} \times \mathbf{R}^{k},(0,0)\right)$ and $H:\left(\mathbf{R}^{p} \times \mathbf{R}^{k},(0,0)\right) \rightarrow$ $\left(\mathbf{R}^{p} \times \mathbf{R}^{k},(0,0)\right)$ such that the diagram $(*)$ in Section 1 commutes, where $\pi:\left(\mathbf{R}^{n} \times\right.$ $\left.\mathbf{R}^{k},(0,0)\right) \rightarrow\left(\mathbf{R}^{k}, 0\right), \pi^{\prime}:\left(\mathbf{R}^{p} \times \mathbf{R}^{k},(0,0)\right) \rightarrow\left(\mathbf{R}^{k}, 0\right)$ are canonical projections.

For given two $C^{\infty}$ map-germs $f, g:\left(\mathbf{R}^{n}, 0\right) \rightarrow\left(\mathbf{R}^{p}, 0\right)$, we consider the following four conditions.

(i) The map-germ $f$ is topologically equivalent to $g$.

(ii) There exist a germ of $C^{\infty}$ diffeomorphism $s:\left(\mathbf{R}^{n}, 0\right) \rightarrow\left(\mathbf{R}^{n}, 0\right)$ and a $C^{\infty}$ map-germ $M:\left(\mathbf{R}^{n}, 0\right) \rightarrow(G L(p, \mathbf{R}), M(0))$ such that the following (a) and (b) are satisfied:

(a) $f(x)=M(x) g(s(x))$,

(b) the $C^{\infty}$ map-germ $F:\left(\mathbf{R}^{n} \times \mathbf{R}^{p},(0,0)\right) \rightarrow\left(\mathbf{R}^{p}, 0\right)$ given by

$$
F(x, \lambda)=f(x)-M(x) \lambda
$$

is a Thom trivial deformation-germ of $f$.

(iii) There exist a germ of $C^{\infty}$ diffeomorphism $s:\left(\mathbf{R}^{n}, 0\right) \rightarrow\left(\mathbf{R}^{n}, 0\right)$ and a $C^{\infty}$ map-germ $M:\left(\mathbf{R}^{n}, 0\right) \rightarrow(G L(p, \mathbf{R}), M(0))$ such that (a), (b) of condition (ii) and the following (c) are satisfied:

(c) The $C^{\infty}$ map-germ $G:\left(\mathbf{R}^{n} \times \mathbf{R}^{p},(0,0)\right) \rightarrow\left(\mathbf{R}^{p}, 0\right)$ given by

$$
G(x, \lambda)=g(x)-M\left(s^{-1}(x)\right)^{-1} \lambda
$$

is a Thom trivial deformation-germ of $g$.

(iv) There exist a germ of $C^{\infty}$ diffeomorphism $s:\left(\mathbf{R}^{n}, 0\right) \rightarrow\left(\mathbf{R}^{n}, 0\right)$ and a $C^{\infty}$ mapgerm $M:\left(\mathbf{R}^{n}, 0\right) \rightarrow(G L(p, \mathbf{R}), M(0))$ such that (a) of condition (ii) and the following (d) are satisfied:

(d) The $C^{\infty}$ map-germ $F:\left(\mathbf{R}^{n} \times \mathbf{R}^{p},(0,0)\right) \rightarrow\left(\mathbf{R}^{p}, 0\right)$ given by

$$
F(x, \lambda)=f(x)-M(x) \lambda
$$

is a transversely Thom trivial deformation-germ of $f$.

Theorem 3.1 ([16]). Let $f, g:\left(\mathbf{R}^{n}, 0\right) \rightarrow\left(\mathbf{R}^{p}, 0\right)$ be $C^{\infty}$ map-germs with rank zero. Then condition (ii) implies condition (i).

Theorem 3.2 ([16]). Condition (iii) implies condition (i).

Theorem 3.3 ([18]). Condition (iv) implies condition (i).

Note that Example 1.1

$$
\begin{aligned}
& f(x, y)=\left(x, y^{3}+x y\right) \\
& g(x, y)=\left(x, y^{3}\right)
\end{aligned}
$$

again shows that condition (ii) does not necessarily imply condition (i) in positive rank case. 
4. The simple systematic method. Let $f:\left(\mathbf{R}^{n}, 0\right) \rightarrow\left(\mathbf{R}^{p}, 0\right)$ be a $C^{\infty}$ map-germ and $\Phi:\left(\mathbf{R}^{n} \times \mathbf{R}^{k},(0,0)\right) \rightarrow\left(\mathbf{R}^{p}, 0\right)$ be a $C^{r}$ deformation-germ of $f$, where $0 \leq r \leq \infty$. Suppose that there exists a $C^{r} \mathcal{A}$-morphism from $\Phi$ to $f$. By the definition of a $C^{r}$ $\mathcal{A}$-morphism, there exist $C^{r}$ map-germs $h:\left(\mathbf{R}^{n} \times \mathbf{R}^{k},(0,0)\right) \rightarrow\left(\mathbf{R}^{n} \times \mathbf{R}^{k},(0,0)\right), H:$ $\left(\mathbf{R}^{p} \times \mathbf{R}^{k},(0,0)\right) \rightarrow\left(\mathbf{R}^{p} \times \mathbf{R}^{k},(0,0)\right)$ and $\varphi:\left(\mathbf{R}^{k}, 0\right) \rightarrow\left(\mathbf{R}^{k}, 0\right)$ such that the following (4.1) and (4.2) hold (for the definition of $C^{r} \mathcal{A}$-morphism, see [17]).

(4.1) For any representatives $\widetilde{h}$ of $h$ and $\widetilde{H}$ of $H$, there exist neighborhoods $U$ of the origin in $\mathbf{R}^{n}, V$ of the origin in $\mathbf{R}^{k}$ and $W$ of the origin in $\mathbf{R}^{p}$ such that the restrictions $\left.\widetilde{h}\right|_{U \times\{\lambda\}}$ and $\left.\widetilde{H}\right|_{W \times\{\lambda\}}$ are $C^{r}$ diffeomorphisms for any $\lambda \in V$.

(4.2) The following diagram commutes, where $\pi:\left(\mathbf{R}^{n} \times \mathbf{R}^{k},(0,0)\right) \rightarrow\left(\mathbf{R}^{k}, 0\right), \pi^{\prime}:$ $\left(\mathbf{R}^{p} \times \mathbf{R}^{k},(0,0)\right) \rightarrow\left(\mathbf{R}^{k}, 0\right)$, are canonical projections:

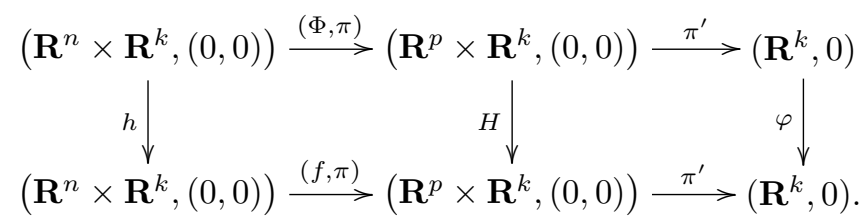

By (4.2), we may write

$$
h(x, \lambda)=\left(h_{1}(x, \lambda), \varphi(\lambda)\right) \quad \text { and } \quad H(y, \lambda)=\left(H_{1}(y, \lambda), \varphi(\lambda)\right) .
$$

Let $\varphi_{H}^{\prime}:\left(\mathbf{R}^{k}, 0\right) \rightarrow\left(\mathbf{R}^{p}, 0\right)$ be the $C^{r}$ map-germ given by

$$
\varphi_{H}^{\prime}(\lambda)=H_{1}(0, \lambda) \text {. }
$$

The map-germ (4.3) is the key idea in our study.

We put also $h^{\prime}:\left(\mathbf{R}^{n} \times \mathbf{R}^{k},(0,0)\right) \rightarrow\left(\mathbf{R}^{n} \times \mathbf{R}^{p},(0,0)\right)$ as

$$
h^{\prime}(x, \lambda)=\left(h_{1}(x, \lambda), \varphi_{H}^{\prime}(\lambda)\right)
$$

and $H^{\prime}:\left(\mathbf{R}^{n} \times \mathbf{R}^{k} \times \mathbf{R}^{p},(0,0,0)\right) \rightarrow\left(\mathbf{R}^{n} \times \mathbf{R}^{p} \times \mathbf{R}^{p},(0,0,0)\right)$ as

$$
H^{\prime}(x, \lambda, y)=\left(h^{\prime}(x, \lambda), H_{1}(y, \lambda)-\varphi_{H}^{\prime}(\lambda)\right) .
$$

Then we can show that $\left\{h^{\prime}, H^{\prime}, \varphi_{H}^{\prime}\right\}$ is a $C^{r} \mathcal{K}$-morphism from $\Phi$ to $F$, where $F$ is the graph deformation-germ of $f$ given by $F(x, y)=f(x)-y$. (For details, see [17]. In [17] the argument is pursued only for $C^{\infty}$ deformation-germs. However, $C^{r}$ deformation-germs can be treated by the exactly parallel argument.)

Next, returning to the situations in Sections 1-3, we let $f, g:\left(\mathbf{R}^{n}, 0\right) \rightarrow\left(\mathbf{R}^{p}, 0\right)$ be $C^{\infty}$ map-germs. We suppose that there exist a $C^{r}$ map-germ $M:\left(\mathbf{R}^{n}, 0\right) \rightarrow(G L(p, \mathbf{R}), M(0))$ and a germ of $C^{r}$ diffeomorphism $s:\left(\mathbf{R}^{n}, 0\right) \rightarrow\left(\mathbf{R}^{n}, 0\right)$ such that $f(x)=M(x) g(s(x))$. We concentrate on considering the following $C^{r}$ deformation-germ of $f$ :

$$
f(x)-M(x) \lambda \text {. }
$$

This deformation-germ is linear with respect to parameter variables. Remark that the parameter space of (4.4) is $p$-dimensional. Thus, if there exists a $C^{r} \mathcal{A}$-morphism $\{h, H, \varphi\}$ from $\Phi$ to $f$, then the map-germ (4.3) is a map-germ between the same dimensional spaces. We suppose furthermore that the deformation-germ (4.4) is $C^{r}$-trivial. Then, there exists 
a $C^{r} \mathcal{A}$-morphism $\{h, H, \varphi\}$ from $\Phi$ to $f$. Thus, from the above argument we see that there exists a $C^{r} \mathcal{K}$-morphism $\left\{h^{\prime}, H^{\prime}, \varphi_{H}^{\prime}\right\}$ from (4.4) to the graph deformation-germ $F$ of $f$. In particular, we have the following equality (for details, see [15], [16]):

$$
f\left(h_{1}(x, g(s(x)))\right)=H_{1}(0, g(s(x))) .
$$

Finally, we can show the following.

Lemma 4.1 ([15], [16]). If the map-germ (4.3) is a germ of $C^{r}$ diffeomorphism, then the endomorphism-germ of $\left(\mathbf{R}^{n}, 0\right)$ given by

$$
x \mapsto h_{1}(x, g(s(x)))
$$

is also a germ of $C^{r}$ diffeomorphism.

Thus, we see that for $0 \leq r \leq \infty$ in order to obtain $C^{r}$ right-left equivalence of $f$ and $g$, it suffices to find that the map-germ (4.3) is a germ of $C^{r}$ diffeomorphism.

\section{Several applications}

5.1. $C^{r}$ right-left equivalence of $C^{r}$-stable map-germs $(1 \leq r \leq \infty)$

Definition 5.1. A $C^{\infty}$ map-germ $f:\left(\mathbf{R}^{n}, 0\right) \rightarrow\left(\mathbf{R}^{p}, 0\right)$ is said to be $C^{r}$-stable if every $C^{\infty}$ deformation-germ of $f$ is $C^{r}$-trivial $(0 \leq r \leq \infty)$.

There are several apparently different definitions of $C^{r}$-stability. For the relation between them, see [20]. Our definition of $C^{r}$-stability is called $P$ - $C^{r}$-stability in [20].

From the argument in Section 4, we have the following (for the definition of $C^{r}$ $\mathcal{K}$-versality, see $[17])$.

TheOREm 5.1 ([17]). For any $C^{r}$-stable map-germ $f$, its graph deformation-germ is $C^{r} \mathcal{K}$-versal for $0 \leq r \leq \infty$.

For $1 \leq r<\infty$, the uniqueness of $C^{r} \mathcal{K}$-versal deformation-germ of a given map-germ may be proved easily by a slight modification of Martinet's proof of the uniqueness of $C^{\infty} \mathcal{K}$-versality (pp. $155-156$ of [1], pp. $21-22$ of [8]), because in order to prove the uniqueness we need only one implication, the $C^{r} \mathcal{K}$-versality implies the infinitesimal $C^{r-1} \mathcal{K}$-versality, which is clear. Thus, by using Martinet's argument (p. 158 of [1], p. 28 of [8]), we see that Theorem 5.1 yields a $C^{r}$ generalization of Mather's classification theorem $(1 \leq r \leq \infty)$ without any difficulty. Note that Theorem 1.2 also yields the same generalization of Mather's classification theorem as a trivial corollary.

Theorem $5.2([15],[17])$. Let $f, g:\left(\mathbf{R}^{n}, 0\right) \rightarrow\left(\mathbf{R}^{p}, 0\right)$ be $C^{r}$-stable map-germs $(1 \leq r \leq \infty)$. Suppose that there exist a germ of $C^{\infty}$ diffeomorphism $s:\left(\mathbf{R}^{n}, 0\right) \rightarrow\left(\mathbf{R}^{n}, 0\right)$ and a $C^{\infty}$ map-germ $M:\left(\mathbf{R}^{n}, 0\right) \rightarrow(G L(p, \mathbf{R}), M(0))$ such that $f(x)=M(x) g(s(x))$. Then $f$ and $g$ are $C^{r}$ right-left equivalent. 


\section{2. $C^{0}$ right-left equivalence of $C^{0}$-stable map-germs}

Definition 5.2. A $C^{\infty}$ map germ $f:\left(\mathbf{R}^{n}, 0\right) \rightarrow\left(\mathbf{R}^{p}, 0\right)$ is said to be $M T$-stable if the jet extension of it is multi-transverse to the Thom-Mather canonical stratification of the jet space.

Concerning $C^{0}$ right-left equivalence of MT-stable map-germs, there is a well-known theorem due to M. Fukuda and T. Fukuda.

Theorem $5.3([3])$. Let $f, g:\left(\mathbf{R}^{n}, 0\right) \rightarrow\left(\mathbf{R}^{p}, 0\right)$ be MT-stable map-germs. Suppose that there exist a germ of $C^{\infty}$ diffeomorphism $s:\left(\mathbf{R}^{n}, 0\right) \rightarrow\left(\mathbf{R}^{n}, 0\right)$ and a $C^{\infty}$ map-germ $M:\left(\mathbf{R}^{n}, 0\right) \rightarrow(G L(p, \mathbf{R}), M(0))$ such that $f(x)=M(x) g(s(x))$. Then, they are $C^{0}$ right-left equivalent.

Definition 5.3. A $C^{\infty}$ map-germ $f:\left(\mathbf{R}^{n}, 0\right) \rightarrow\left(\mathbf{R}^{p}, 0\right)$ is said to be Thom stable if every $C^{\infty}$ deformation-germ of $f$ is Thom trivial.

As a consequence of the definition of MT-stability, every $C^{\infty}$ deformation-germ of an MT-stable map-germ is Thom trivial (see [7], [11]]). Thus, every MT-stable map-germ is Thom stable. By Thom's second isotopy lemma ([2], [7], [11]), every Thom stable mapgerm is $C^{0}$-stable in the sense of Definition 5.1. As a trivial corollary of Theorem 3.2, we obtain a generalization of Theorem 5.3.

TheOREM 5.4 ([16]). Let $f, g:\left(\mathbf{R}^{n}, 0\right) \rightarrow\left(\mathbf{R}^{p}, 0\right)$ be Thom stable map-germs. Suppose that there exist a germ of $C^{\infty}$ diffeomorphism $s:\left(\mathbf{R}^{n}, 0\right) \rightarrow\left(\mathbf{R}^{n}, 0\right)$ and a $C^{\infty}$ map-germ $M:\left(\mathbf{R}^{n}, 0\right) \rightarrow(G L(p, \mathbf{R}), M(0))$ such that

$$
f(x)=M(x) g(s(x)) .
$$

Then, they are $C^{0}$ right-left equivalent.

5.3. An estimate of the order of $C^{\infty}$ determinacy. As an application of Theorem 2.3, we show the following.

Theorem 5.5. Let $f:\left(\mathbf{R}^{n}, 0\right) \rightarrow\left(\mathbf{R}^{p}, 0\right)$ be a $C^{\infty}$ map-germ. Suppose that there exist positive integers $k, \ell$ such that

(a) $\quad m_{n}^{k} \theta(f) \subset T \mathcal{A}(f) \quad$ and

(b) $\quad m_{n}^{\ell} \theta(f) \subset T \mathcal{K}(f)$.

Then, $f$ is $(k+\ell-1)$-determined with respect to $C^{\infty}$ right-left equivalence.

The set $T \mathcal{K}(f)$ is defined by

$$
T \mathcal{K}(f)=t f\left(m_{n} \theta(n)\right)+f^{*} m_{p} \theta(f)
$$

where $f^{*}(u)=u \circ f$. This set is the tangent space of the orbit through $f$ by the action of the group $\mathcal{K}$ which was introduced by Mather in [9]. For details on the group $\mathcal{K}, T \mathcal{K}(f)$ and the definition of determinacy, see [9], [21]. Theorem 5.5 is a similar estimate to the well-known estimate due to Gaffney ([4]). Theorem 5.5 has been stated already in [13] without the proof. Several applications of Theorem 5.5 to divergent diagrams have been obtained in [13]. 
Proof. Let ${ }_{k-1} \mathcal{K}$ be the set of all pairs of $(s, M)$, where $s$ is a germ of $C^{\infty}$ diffeomorphism $\left(\mathbf{R}^{n}, 0\right) \rightarrow\left(\mathbf{R}^{n}, 0\right)$ and $M:\left(\mathbf{R}^{n}, 0\right) \rightarrow\left(G L(p, \mathbf{R}), E_{p}\right)$ is a $C^{\infty}$ map-germ with each entry of $M-M(0)$ belonging to $m_{n}^{k}$. Here, $E_{p}$ is the $p$ by $p$ unit matrix. The set ${ }_{k-1} \mathcal{K}$ is a group by the operation $\left(s_{1}, M_{1}\right) *\left(s_{2}, M_{2}\right)=\left(s_{1} \circ s_{2}, M_{1} M_{2}\right)$, where $s_{1} \circ s_{2}$ is the composition of $s_{1}$ and $s_{2}$ and $M_{1} M_{2}$ is the product of matrices of $M_{1}$ and $M_{2}$. The group $_{k-1} \mathcal{K}$ is a subgroup of the group $\mathcal{K}$ and the tangent space of the orbit through $f$ by the action of the group ${ }_{k-1} \mathcal{K}$ is

$$
T_{k-1} \mathcal{K}(f)=t f\left(m_{n} \theta(n)\right)+f^{*} m_{p} m_{n}^{k} \theta(f) .
$$

Condition (b) of Theorem 5.5 implies

$$
m_{n}^{k+\ell} \theta(f) \subset t f\left(m_{n}^{k+1} \theta(n)\right)+f^{*} m_{p} m_{n}^{k} \theta(f) \subset T_{k-1} \mathcal{K}(f) .
$$

By (5.3.2), we see that $f$ is $(k+\ell)$-determined with respect to the group ${ }_{k-1} \mathcal{K}$.

Let $g:\left(\mathbf{R}^{n}, 0\right) \rightarrow\left(\mathbf{R}^{p}, 0\right)$ be a $C^{\infty}$ map-germ with $j^{k+\ell-1} f(0)=j^{k+\ell-1} g(0)$. Then, since $k>0,(5.3 .2)$ implies

$$
m_{n}^{k+\ell} \theta(g) \subset t g\left(m_{n}^{k+1} \theta(n)\right)+g^{*} m_{p} m_{n}^{k} \theta(g)+m_{n}^{k+\ell+1} \theta(g) .
$$

By Mather's lemma (Lemma 3.1 of [10]), (5.3.3) implies that there exist a germ of $C^{\infty}$ diffeomorphism $s:\left(\mathbf{R}^{n}, 0\right) \rightarrow\left(\mathbf{R}^{n}, 0\right)$ and a $C^{\infty}$ map-germ $M:\left(\mathbf{R}^{n}, 0\right) \rightarrow\left(G L(p, \mathbf{R}), E_{p}\right)$ such that

$$
\text { (5.3.5) each entry of } M-M(0) \text { belongs to } m_{n}^{k} \text {. }
$$

By Theorem 2.3, (5.3.4), (5.3.5) and condition (a) of Theorem 5.5 imply that $f$ and $g$ are $C^{\infty}$ right-left equivalent.

Remark 5.1. Note that in the proof of Theorem 5.5 we use Mather's lemma only for orbits by ${ }_{k-1} \mathcal{K}$ group action, whose tangent spaces are much simpler than $T \mathcal{A}(g)$.

Since $T \mathcal{A}(f) \subset T \mathcal{K}(f)$, Theorem 5.5 yields the following well-known estimate due to du Plessis and Wall as a trivial corollary.

Theorem 5.6 ([19], [21]). Let $f:\left(\mathbf{R}^{n}, 0\right) \rightarrow\left(\mathbf{R}^{p}, 0\right)$ be a $C^{\infty}$ map-germ. Suppose that there exists a positive integer $k$ such that

$$
m_{n}^{k} \theta(f) \subset T \mathcal{A}(f) .
$$

Then, $f$ is $(2 k-1)$-determined with respect to $C^{\infty}$ right-left equivalence.

\section{References}

[1] V. I. Arnol'd, S. M. Guse ̌n-Zade, A. N. Varchenko, Singularities of Differentiable Maps I, Monogr. Math. 82, Birkhäuser, Boston, 1985.

[2] K. Bekka, C-régularité et trivialité topologique, in: Singularity Theory and its Applications (Warwick, 1989), Part I, D. Mond and J. Montaldi (eds.), Lecture Notes in Math. 1462, Springer, Berlin, 1991, 42-62. 
[3] M. Fukuda and T. Fukuda, Algebras $Q(f)$ determine the topological types of generic map germs, Invent. Math. 51 (1979), 231-237.

[4] T. Gaffney, A note on the order of determination of a finitely determined germ, Invent. Math. 52 (1979), 127-130.

[5] T. Gaffney, The structure of $T \mathcal{A}(f)$, classification and an application to differential geometry, Proc. Sympos. Pure Math. 40 (1983), 409-427.

[6] T. Gaffney and A. A. du Plessis, More on the determinacy of smooth map-germs, Invent. Math. 66 (1982), 137-163.

[7] C. G. Gibson, K. Wirthmüller, A. A. du Plessis, E. J. N. Looijenga, Topological Stability of Smooth Mappings, Lecture Notes in Math. 552, Springer, Berlin, 1976.

[8] J. Martinet, Déploiements versals des applications différentiables et classification des applications stables, in: Singularités d'applications différentiables (Plans-sur-Bex, 1975), O. Burlet, F. Ronga (eds.), Lecture Notes in Math. 535, Springer, Berlin, 1976, 1-44.

[9] J. Mather, Stability of $C^{\infty}$ mappings, III. Finitely determined map-germs, Inst. Hautes Études Sci. Publ. Math. 35 (1968), 127-156.

[10] J. Mather, Stability of $C^{\infty}$ mappings, IV. Classification of stable map-germs by R-algebras, Inst. Hautes Études Sci. Publ. Math. 37 (1969), 223-248.

[11] J. Mather, How to stratify mappings and jet spaces, in: Singularités d'applications différentiables (Plans-sur-Bex, 1975), O. Burlet, F. Ronga (eds.), Lecture Notes in Math. 535, Springer, Berlin, 1976, 128-176.

[12] D. Mond, On the classification of germs of maps from $\mathbf{R}^{2}$ to $\mathbf{R}^{3}$, Proc. London Math. Soc. (3) 50 (1985), 333-369.

[13] T. Nishimura, A constructive method to get right-left equivalence for smooth map germs and its application to divergent diagrams, in: Workshop on Real and Complex Singularities (São Carlos, 1992), M. A. S. Ruas (ed.), Mat. Contemp. 5, Sociedade Brasileira de Matemática, Rio de Janeiro, 1993, 137-160.

[14] T. Nishimura, Isomorphism of smooth map germs with isomorphic local algebras, in: Real Analytic and Algebraic Singularities (Nagoya, 1996), T. Fukuda, T. Fukui, S. Izumiya and S. Koike (eds.), Pitman Res. Notes Math. Ser. 381, Longman, Harlow, 1998, 94-106.

[15] T. Nishimura, Criteria for right-left equivalence of smooth map-germs, preprint, Yokohama National University, 1998.

[16] T. Nishimura, Topological equivalence of $\mathcal{K}$-equivalent map germs, J. London Math. Soc. (2), to appear.

[17] T. Nishimura, $C^{r} \mathcal{K}$-versality of the graph deformation of a $C^{r}$ stable map-germ, Math. Proc. Cambridge Philos. Soc., to appear.

[18] T. Nishimura, Topological equivalence of $\mathcal{K}$-equivalent map germs, II, in preparation.

[19] A. A. du Plessis, On the determinacy of smooth map-germs, Invent. Math. 58 (1980), 107-160.

[20] A. A. du Plessis and C. T. C. Wall, The Geometry of Topological Stability, London Math. Soc. Monogr. (N.S.) 9, Oxford University Press, New York, 1995.

[21] C. T. C. Wall, Finite determinacy of smooth map-germs, Bull. London Math. Soc. 13 (1981), 481-539. 